\title{
Spotted around the web: Parental age, autism-schizophrenia overlap, bumetanide trials
}

\author{
BY JILL ADAMS, ANGIE VOYLES ASKHAM, LAURA DATTARO, PETER HESS
}

24 SEPTEMBER 2021

WEEK OF SEPTEMBER $20^{\mathrm{TH}}$

\section{Research roundup}

- Women with autistic children had significantly lower blood levels of an immune molecule called IL-17A during pregnancy than did those with non-autistic children. Journal of Autism and Developmental Disorders

- Young autistic adults' vocational activities do not seem to change from age 18 to 28. Autism Research

- Gene variants that occur in both autism and schizophrenia may share similar functional aspects. Nature Communications

- Variants in the autism-linked gene SCN2A that are associated with reduced sodium channel function sometimes lead to increased neuronal excitability, which may help to explain treatment-resistant seizures. Spectrum covered similar research in August. Cell Reports

- Older maternal and paternal ages at conception are associated with having an autistic child, according to a study in Oman. Spectrum has an explainer on parental age and autism. Sultan Qaboos University Medical Journal

- A mutation in TRIO, an autism-linked gene, that prevents its protein from interacting with NLGN1 seems to affect the formation of glutamate synapses. Journal of Neuroscience

- Few pediatricians in Israel say they are trained well enough to diagnose and manage children with developmental disabilities. Israel Journal of Health Policy Research

- Autistic and non-autistic adults say that stimming helps them manage sensory sensitivity and emotions, but that they feel social pressure to suppress the behavior. Spectrum has reported on autistic adults' efforts to reframe the conversation around repetitive behaviors. Research in Autism Spectrum Disorders 


\section{Spectrum | Autism Research News}

https://www.spectrumnews.org

- Knocking out the autism-linked gene MEF2C in mice disrupts the development of some types of interneurons more than others. Nature

- Researchers from Brazil argue that people with autism should be included in priority groups for coronavirus vaccination, along with the elderly, healthcare workers and those with medical conditions that put them at risk of COVID-19. Journal of Autism and Developmental Disorders

- The pharmaceutical companies Servier and Neurochlore have terminated their phase 3 trials of bumetanide because early results showed a lack of efficacy. The Pharma Letter

\section{Science and society}

- Movies with autistic characters tend to feature the characters' exceptional abilities and do not represent the full spectrum of autism. The Guardian

- A Wisconsin college has denied a request to teach online from a professor on the spectrum who struggles to wear a mask. Wisconsin State Journal

- The coronavirus pandemic has contributed to burnout in many autistic people. Spectrum has covered autistic burnout in an explainer. The New York Times

- One in five caregivers give their autistic child cannabidiol to treat a suite of issues, including anxiety, sleep problems and challenging behavior, according to a survey. Cannigma

Cite this article: https://doi.org/10.53053/LNXD6831 